\title{
STING signaling is a potential immunotherapeutic target in colorectal cancer
}

\author{
Hong Jae Chon ${ }^{1,2,5^{*}}$, Hyojoong Kim ${ }^{1,2^{*}}$, Jung Hyun Noh ${ }^{1,5^{*}}$, Hannah Yang1,2, Won Suk Lee ${ }^{1,2}$, So Jung Kong ${ }^{1,2}$, \\ Seung Jun Lee ${ }^{1,2}$, Yu Seong Lee 1,2, Woo Ram Kim³ ${ }^{3}$ Joo Hang Kim¹, Gwangil Kim ${ }^{4,5}$, Chan Kim ${ }^{1,2,5 凶}$ \\ 1. Medical Oncology, CHA Bundang Medical Center, Seongnam, Korea \\ 2. Laboratory of Translational Immuno-Oncology, Seongnam, Korea \\ 3. Department of Surgery, CHA Bundang Medical Center, Seongnam, Korea \\ 4. Department of Pathology, CHA Bundang Medical Center, Seongnam, Korea \\ 5. CHA Medical School, CHA University, Seongnam, Korea \\ * These authors equally contributed this work.
}

$\triangle$ Corresponding authors: Chan Kim, Medical Oncology, Department of Internal Medicine, CHA Bundang Medical Center, 59 Yatap-ro, Bundang-gu, Seongnam, 13496, Korea; E-mail: chan@cha.ac.kr or Gwangil Kim, Department of Pathology, CHA Bundang Medical Center, 59 Yatap-ro, Bundang-gu, Seongnam, 13496, Korea; E-mail: blacknw@cha.ac.kr

(c) The author(s). This is an open access article distributed under the terms of the Creative Commons Attribution License (https://creativecommons.org/licenses/by/4.0/). See http://ivyspring.com/terms for full terms and conditions.

Received: 2019.01.04; Accepted: 2019.07.22; Published: 2019.08.27

\begin{abstract}
Background: Stimulator of Interferon Genes (STING) is an innate immune sensor for cytosolic DNA. STING signaling activation is indispensable for type I interferon response and the anti-cancer immune response by CD8 ${ }^{+} \mathrm{T}$ cells. The aim of this study was to characterize intratumoral STING expression pattern and its clinical implication in colorectal cancer (CRC).

Methods: We analyzed STING and CD8 expression in 225 CRC patients who underwent surgical resection. Clinicopathological variables and survival outcomes were analyzed according to STING expression levels. Mice with syngeneic MC38 tumors were also treated with a STING agonist, and tumor microenvironments were analyzed using immunofluorescent staining and flow cytometry.

Results: Distinct STING expression was observed in the CRC tumor specimens. Patients with higher STING expression had early stage cancer with increased intratumoral CD8 ${ }^{+} \mathrm{T}$ cell infiltration and less frequent lymphovascular invasion. Compared to CRC patients with lower STING expression, those with higher STING expression had longer overall and recurrence-free survival. Multivariate Cox regression model also revealed higher STING expression to be an independent prognostic factor for better overall survival. When MC38 colon tumors were treated with intratumoral injection of STING agonist, tumor growth was remarkably suppressed with increased intratumoral $\mathrm{CD}^{+} \mathrm{T}$ cell infiltration. Moreover, T-cell activation markers, ICOS and IFN- $\gamma$, were also upregulated in $\mathrm{CD}^{+} \mathrm{T}$ cells, indicating enhanced effector $\mathrm{T}$ cell function after STING treatment.

Conclusion: We confirmed the distinct STING expression in CRC and demonstrated its independent prognostic value in survival outcomes. STING could be a potential therapeutic target that enhances anti-cancer immune response in CRC.
\end{abstract}

Key words: STING; Colorectal cancer; Prognosis; Immunotherapy; Inflamed tumor

\section{Introduction}

Colorectal cancer $(\mathrm{CRC})$ remains the third most common cancer worldwide, as over two million patients were newly diagnosed annually and more than one million died of CRC $[1,2]$. Although the rates of CRC death are dropping in recent decades, the 5 -year survival rate was only $13 \%$ in stage IV CRCs. Therefore, a CRC is a life-threatening malignancy with a high demand for effective treatment, especially when diagnosed at an advanced stage [3]. Over the last decade, advances in systemic chemotherapy and the introduction of the 'continuum of care' strategy have made remarkable progress on CRC treatment. However, the biologic heterogeneity of CRC among patients still results in discrepancies in 
treatment response and survival outcome, which makes it harder to treat CRC $[4,5]$.

The immune system is essential for detecting and eliminating cancer cells, and adaptive anti-cancer immune responses driven by effector $\mathrm{T}$ cells are especially indispensable in the immune surveillance of cancer [6-8]. Since this immunologic monitoring is defective in many human malignancies, immunotherapeutic agents that can potently augment effector $\mathrm{T}$ cell function against cancer are being developed and actively introduced into clinical practice recently [7, 9]. However, the therapeutic efficacy of cancer immunotherapy in CRC is severely hampered due to the poorly-immunogenic tumor cells and immunosuppressive tumor microenvironment [10-12]. Therefore, a better understanding of the immunologic features of CRC and identification of novel immune targets are necessary to overcome these obstacles and elicit optimal immunity against CRC.

Stimulator of Interferon Genes (STING), an adaptor transmembrane protein localized in the endoplasmic reticulum, is a vital innate immune sensor that detects tumor-derived DNA[13-15]. The activation of the STING pathway induces a robust type I interferon (IFN) production, followed by activation of dendritic cells for the cross-priming of $T$ cells, and elicitation of an adaptive immune response against tumors [15-17]. Recent studies illustrated that STING is expressed in various human malignancies including melanomas, gastric cancer, and hepatocellular carcinoma, and it is correlated with $\mathrm{T}$ cell-mediated cancer immunity and the prognosis of those cancers [14, 18-20]. Although the exact function of STING in human CRC has not been fully elucidated, the potential of STING in CRC has been strongly suggested in many animal studies, where it was found to mediate protection against CRC carcinogenesis [17, 21-23].

In this study, we aimed to explore the clinical value of STING as a prognostic immune biomarker in CRC patients and to evaluate its potential as an immunotherapeutic target in CRC.

\section{Materials and Methods}

\section{Patients and tissue samples}

This study was performed retrospectively on patients diagnosed with CRC at the CHA Bundang Medical Center (Seongnam, Korea) from 2002 to 2006. Tumor samples from 225 CRC patients were examined for STING and CD8 expression. The clinicopathological characteristics, such as gender, age, tumor location, differentiation, growth, stage, lymphovascular invasion (LVI), perineural invasion
(PNI), microsatellite status (MSI), history of adjuvant therapy, recurrence, and survival outcome, were obtained from the electronic medical records at the institute. The $7^{\text {th }}$ edition of the American Joint Committee on Cancer guideline for tumor, node, and metastasis (TNM) classification was used for staging. The study was approved by the institutional review committee (IRB File No. 2017-11-054).

\section{Tissue microarray (TMA) construction and histologic analysis}

Simple and precise paraffin TMAs were constructed using a conventional micro-compound table and a drill grinder. The original hematoxylin and eosin (H\&E) slides were observed by pathologists. Two different tumor areas per case were selected for TMA construction. Core tissue biopsies, with a diameter of $3 \mathrm{~mm}$, were taken from the individual paraffin blocks as donor blocks and arranged into tissue array blocks as recipient paraffin blocks using a trephine apparatus. All TMA blocks were stained with $\mathrm{H} \& \mathrm{E}$ for confirmation.

TMA blocks from CRC patients were cut into 5 $\mu \mathrm{m}$-thick sections and immunohistochemical staining was performed using anti-STING (rabbit, clone D1V5L, Cell Signaling) or anti-CD8 (rabbit, clone SP57, Roche) antibodies. The BenchMark XT (Ventana) with heat-induced epitope retrieval (CC1 solution, Ventana) and the iView DAB detection kit (Ventana) was used as the visualization system. After the slides were mounted, high-resolution digital images of whole slides were taken with a BX43 microscope (Olympus). Immunofluorescent staining was performed on cryosectioned mouse tumor tissues with anti-CD31 (hamster, clone 2H8, Millipore) and anti-CD8 (rat, clone 53-6.7, BD Pharmingen) antibodies as previously described [8, 24]. Immunofluorescent images were acquired with a LSM 880 confocal microscope (Zeiss).

Density measurement of $\mathrm{STING}^{+}$or $\mathrm{CD}^{+}$areas was performed with the ImageJ software running the Fiji image processing package (https://imagej.net/ Fiji). The color channels with hematoxylin and diaminobenzidine were separated and quantified to determine immunoreactive areas. Automated counting was applied for the analysis of all images. STING expression was assessed both in tumor and immune cells. The cut-off values to define high or low expression of STING or CD8 were the median values of all samples which were determined with ImageJ software.

\section{Mice and cell line}

Male C57BL/6 mice (8 weeks old) were purchased from Orient Bio Inc. (Seongnam, Korea) 
and housed in a specific pathogen-free animal facility at CHA University (Seongnam, Korea). All experiments were approved by the Institutional Animal Care and Use Committee of CHA University (IACUC 170168). The MC38 murine colon cancer cell line was obtained from the National Cancer Center (Goyang, Korea). They were cultured in Dulbecco's Modified Eagle Medium supplemented with 10\% fetal bovine serum (FBS) and 1\% penicillin/streptomycin, and maintained at $37^{\circ} \mathrm{C}$ in a $5 \% \mathrm{CO}_{2}$ incubator.

\section{Tumor model and treatment}

All C57BL/ 6 mice were subcutaneously injected with $1 \times 10^{6}$ MC38 cells on the right flank. When tumors reached $>5 \mathrm{~mm}$ in diameter, a STING agonist, 3'3'-cGAMP (10 $\mu \mathrm{g}$ in $50 \mu \mathrm{L}$ of PBS; Invivogen), was intratumorally injected at D7, D10, and D13 after tumor implantation. Tumor volumes were measured using a digital caliper and calculated using the formula $1 / 2 \times A \times B^{2}$, where $A$ is the longest diameter and $\mathrm{B}$ is its perpendicular diameter.

\section{Flow Cytometry Analysis}

Tumors were harvested and chopped into several pieces. The tumor pieces were digested into single cell suspensions by incubating in digestion buffer $[2 \mathrm{mg} / \mathrm{mL}$ collagenase $\mathrm{D}$ (Merck) and 40 $\mu \mathrm{g} / \mathrm{mL}$ DNase I (Merck)] for $1 \mathrm{~h}$ at $37{ }^{\circ} \mathrm{C}$. Cell suspensions were filtered using a $70-\mu \mathrm{m}$ cell strainer (Corning) and a 40- $\mathrm{mm}$ nylon mesh to remove cell clumps. After washing with FACS buffer (1\% FBS in PBS), cells were primed with antibodies targeting CD45 (30-F11, BD Pharmingen), CD4 (RM4-5, BD Pharmingen), CD8 (53-6.7, BD Pharmingen), CD3 (17A2, eBioscience), or ICOS (7E.17G9, eBioscience). Next, the cells were permeabilized using a permeabilization kit (eBioscience) and stained for IFN- $\gamma$ (eBioscience). Data was acquired using a CytoFLEX flow cytometer (Beckman Coulter) and analyzed using the FlowJo software (Tree Star Inc., Ashland, OR, USA).

\section{Statistical analysis}

Statistical analysis was performed using SPSS version 18.0 (IBM Corporation, Chicago, IL, USA). The correlation between STING expression and the clinicopathological variables was analyzed using the independent sample $t$-test for the continuous variables and the chi-square test for the discrete variables. In survival analysis, recurrence-free survival (RFS) was defined as the time interval between surgery and tumor recurrence or last follow-up. Overall survival (OS) was defined as the time interval from diagnosis to death or last follow-up. Kaplan-Meier method along with the log-rank test was used for survival analyses. The relationship between OS and the clinicopathological features was assessed using multivariate Cox proportional-hazards model. $\mathrm{P}<0.05$ was considered to be statistically significant.

\section{Results}

\section{Baseline patient characteristics}

The baseline clinicopathological characteristics are shown in Table 1 . The male-to-female ratio was $1.25: 1$, and the mean age at diagnosis was 62 years. While $41 \%$ of the tumors were located in the rectum, the others were found in the colon. Most (85\%) tumors were well- or moderately-differentiated tubular adenocarcinoma. LVI was present in $33 \%$ and PNI was present in $9 \%$ of the patients. All patients underwent surgical resection of the primary tumors. Distant metastasis was present in $8 \%$ of the patients at the time of diagnosis.

Table 1. Clinicopathological characteristics according to STING expression

\begin{tabular}{|c|c|c|c|c|c|}
\hline \multicolumn{2}{|l|}{ Factors } & $\begin{array}{l}\text { All } \\
\text { patients } \\
(n=225)\end{array}$ & $\begin{array}{l}\begin{array}{l}\text { STING-low } \\
(\mathrm{n}=112)\end{array} \\
\mathrm{N}(\%)\end{array}$ & $\begin{array}{l}\begin{array}{l}\text { STING-high } \\
(\mathrm{n}=113)\end{array} \\
\mathrm{N}(\%)\end{array}$ & P-value \\
\hline \multirow{3}{*}{ Sex } & & & & & \\
\hline & Female & $100(44.4)$ & $52(46.4)$ & $48(42.5)$ & 0.551 \\
\hline & Male & $125(55.6)$ & $60(53.6)$ & $65(57.5)$ & \\
\hline Age (mean) & & $62.0 \pm 12.1$ & 61.6 & 61.6 & 0.976 \\
\hline \multirow[t]{4}{*}{ Location } & Right & $36(1.0)$ & $19(17.0)$ & $17(15.0)$ & 0.520 \\
\hline & Transverse & $14(6.2)$ & $7(6.3)$ & $7(6.2)$ & \\
\hline & Left & $83(36.9)$ & $36(32.1)$ & 47 (41.6) & \\
\hline & Rectum & $92(40.9)$ & $50(44.6)$ & $42(37.2)$ & \\
\hline \multirow[t]{4}{*}{ Histology } & WD & $14(6.2)$ & $7(6.3)$ & $7(6.2)$ & 0.267 \\
\hline & MD & 177 (78.7) & $91(81.3)$ & $86(76.1)$ & \\
\hline & PD & $13(5.8)$ & $3(2.7)$ & $10(8.8)$ & \\
\hline & Mucinous & $21(9.3)$ & $11(9.8)$ & $10(8.8)$ & \\
\hline \multirow[t]{3}{*}{ Growth } & Fungating & $75(33.3)$ & $38(33.9)$ & $37(32.7)$ & 0.801 \\
\hline & Ulcerofungating & $67(29.8)$ & 35 (31.3) & $32(28.3)$ & \\
\hline & Ulceroinfiltrative & $83(36.9)$ & $39(34.8)$ & $44(38.9)$ & \\
\hline \multirow[t]{4}{*}{$\mathbf{T}$} & $\mathrm{T} 1$ & $2(0.9)$ & $1(0.9)$ & $1(0.9)$ & 0.061 \\
\hline & $\mathrm{T} 2$ & $21(9.3)$ & $5(4.5)$ & $16(14.2)$ & \\
\hline & $\mathrm{T} 3$ & $162(72.0)$ & $82(73.2)$ & $80(70.8)$ & \\
\hline & $\mathrm{T} 4$ & $40(17.8)$ & $24(21.4)$ & $16(14.2)$ & \\
\hline \multirow[t]{3}{*}{$\mathbf{N}$} & No & $109(48.4)$ & $42(37.5)$ & $67(59.3)$ & 0.004 \\
\hline & N1 & $60(26.7)$ & $35(31.3)$ & $25(22.1)$ & \\
\hline & N2 & $56(24.9)$ & 35 (31.3) & $21(18.6)$ & \\
\hline \multirow[t]{4}{*}{ Stage } & I & $19(8.4)$ & $4(3.6)$ & 15 (13.3) & 0.001 \\
\hline & II & 88 (39.1) & $36(32.1)$ & $52(46.0)$ & \\
\hline & III & $100(44.4)$ & $58(51.8)$ & $42(37.2)$ & \\
\hline & IV & $18(8.0)$ & 14 (12.5) & $4(3.5)$ & \\
\hline \multirow[t]{2}{*}{ CD8 } & Low & $113(50.2)$ & $72(64.3)$ & $41(36.3)$ & $<0.001$ \\
\hline & High & $112(49.8)$ & 40 (35.7) & $72(63.7)$ & \\
\hline \multirow[t]{2}{*}{ LVI } & No & $151(67.1)$ & $67(59.8)$ & $84(74.3)$ & 0.020 \\
\hline & Yes & $74(32.9)$ & 45 (40.2) & $29(25.7)$ & \\
\hline \multirow[t]{2}{*}{ PNI } & No & $204(90.7)$ & $102(91.1)$ & $102(90.3)$ & 0.835 \\
\hline & Yes & $21(9.3)$ & $10(8.9)$ & $11(9.7)$ & \\
\hline \multirow[t]{3}{*}{ MSI } & Stable & $137(81.1)$ & $63(81.8)$ & $74(80.4)$ & 0.621 \\
\hline & MSI-low & $13(7.7)$ & $7(9.1)$ & $6(6.5)$ & \\
\hline & MSI-high & $19(11.2)$ & $7(9.1)$ & $12(13.0)$ & \\
\hline Adjuvant & No & $103(45.8)$ & 45 (43.7) & $58(56.3)$ & 0.093 \\
\hline Chemotherapy & Yes & $122(54.2)$ & $67(54.9)$ & $55(45.1)$ & \\
\hline Adjuvant & No & $194(86.2)$ & $95(84.8)$ & 99 (87.6) & 0.544 \\
\hline Radiotherapy & Yes & $31(13.8)$ & 17 (15.2) & $14(12.4)$ & \\
\hline $\begin{array}{l}\text { WD, well differe } \\
\text { differentiated; L } \\
\text { Microsatellite In }\end{array}$ & $\begin{array}{l}\text { entiated; MD, moc } \\
\text { CVI, lymphovascul } \\
\text { nstability. }\end{array}$ & leratedly & erentiated & poorly & SI, \\
\hline
\end{tabular}


A

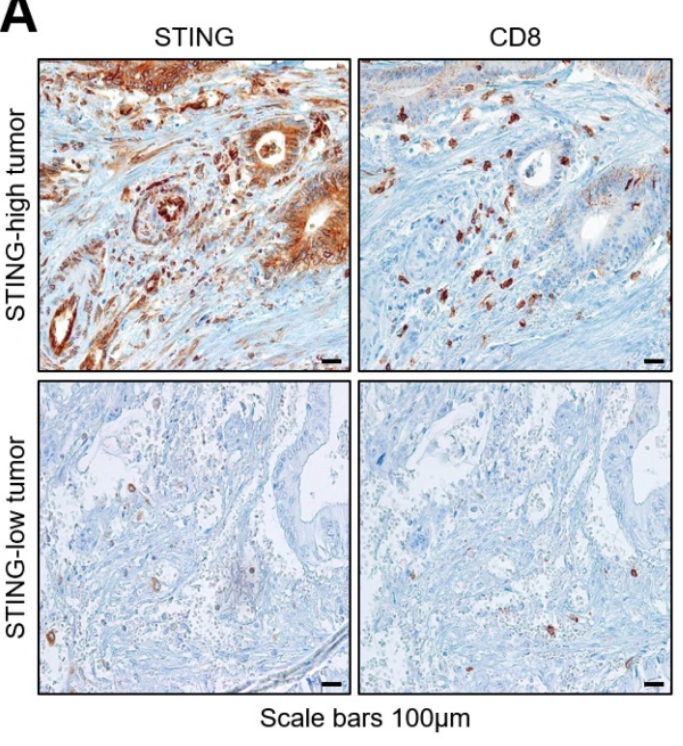

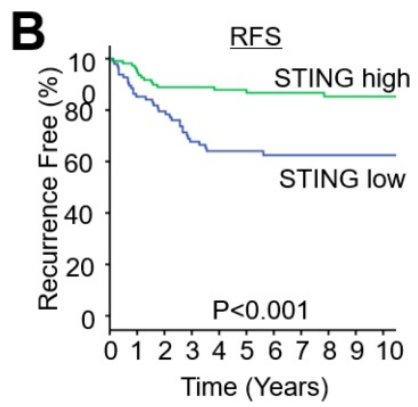

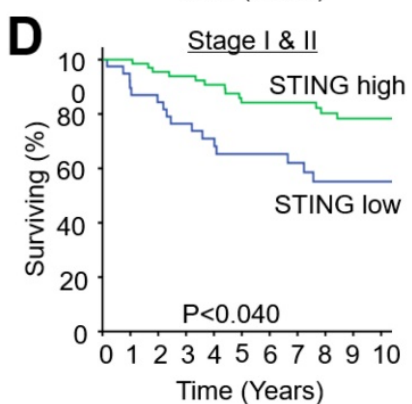

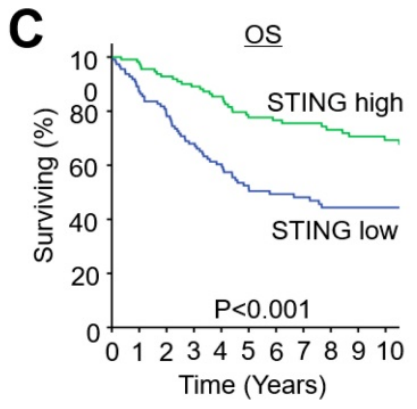

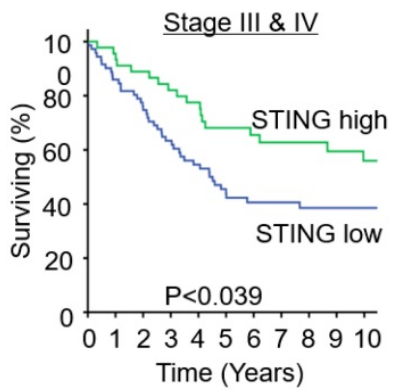

Figure 1. STING expression and the prognosis of CRC according to STING expression. (A) Representative images of STING and CD8 expression in CRCs. (B) Survival curves for recurrence-free survival (RFS). (C) Survival curves for overall survival (OS). (D) Survival curves for OS in early (stage I and II) and late (stage III and IV) stage tumors.

\section{STING expression and clinicopathological characteristics}

CRC tumor tissues were immunostained to visualize STING or CD8 expression (Figure 1A). Representative images of STING-high or -low tumors are shown in Figure 1A. The median of STING expression level in all samples was used as a cut-off value. STING is expressed in the cytoplasm of tumor cells, immune cells, and stromal cells. Notably, tumors with high STING expression had high intratumoral infiltration of $\mathrm{CD}^{+} \mathrm{T}$ cells.

Table 1 summarizes the correlation between clinicopathological characteristics and the level of STING expression. High STING expression significantly correlated with tumor stage (especially $\mathrm{N}$ ) and intratumoral $\mathrm{CD}^{+} \mathrm{T}$ cells. No significant difference in STING expression was observed with respect to sex, age, tumor location, histology, growth type, PNI, MSI statue, and history of adjuvant therapy.

\section{STING expression and survival analysis}

After a median follow-up of 74 months, 97 patients were found to have died at the time of survival analysis. The Kaplan-Meier survival analyses showed that patients with high STING expression had longer RFS when compared to those with low STING expression (5-year RFS rate, $85.3 \%$ vs. $62.5 \%$; $\mathrm{P}<$ 0.001 , Figure 1B). The same was also true for OS (5-year OS rate, $77.7 \%$ vs. $51.5 \%$; P < 0.001, Figure 1C). When the OS of CRC patients was analyzed according to their stage, patients at the early stage $(\mathrm{I}+\mathrm{II})$ or advanced stage (III + IV) showed longer OS when they exhibited high STING expression (Figure 1D).

The clinicopathologic parameters significantly correlated with OS using univariate analyses were age $(P=0.047), T$ stage $(P=0.030), N$ stage $(P<0.001)$, CD8 expression $(\mathrm{P}=0.009)$, STING expression $(\mathrm{P}<$ $0.001)$, presence of LVI $(\mathrm{P}<0.001)$, and presence of PNI ( $\mathrm{P}=0.021)$ (Table 2). When multivariate Cox regression analysis was conducted with these variables, age $(\mathrm{P}=0.009)$, STING expression $(\mathrm{P}=$ $0.012)$, and the presence of LVI remained as independent prognostic factors for OS in CRC patients $(P=0.001)$. Thus, it seems that high STING expression is a better prognostic factor than CD8 for OS in CRC patients both in univariate and multivariate analyses.

Table 2. Univariate and multivariate analyses for overall survival

\begin{tabular}{|c|c|c|c|c|}
\hline \multirow[t]{2}{*}{ Variables } & & \multirow{2}{*}{$\frac{\text { Univariate }}{\text { P-value }}$} & \multicolumn{2}{|l|}{ Multivariate } \\
\hline & & & HR (95 CI) & P-value \\
\hline Sex & (Male vs. Female) & 0.875 & & \\
\hline Age & ( $\geq 65$ vs. $<65)$ & 0.047 & $1.733(1.150-2.612)$ & 0.009 \\
\hline Location & $\begin{array}{l}\text { (Rectum vs. } \\
\text { Colon) }\end{array}$ & 0.567 & & \\
\hline Histology & $\begin{array}{l}\text { (WMD vs. } \\
\text { Others) }\end{array}$ & 0.333 & & \\
\hline $\mathrm{T}$ & (T3,T4 vs. T1,T2) & 0.030 & $1.670(0.711-3.925)$ & 0.239 \\
\hline $\mathrm{N}$ & (N1,N2 vs. N0) & $<0.001$ & $1.531(0.981-2.391)$ & 0.061 \\
\hline $\begin{array}{l}\text { Distant } \\
\text { metastasis }\end{array}$ & (M1 vs M0) & 0.059 & & \\
\hline CD8 & (High vs. Low) & 0.009 & $0.825(0.536-1.270)$ & 0.382 \\
\hline STING & (High vs. Low) & $<0.001$ & $0.573(0.370-0.886)$ & 0.012 \\
\hline LVI & (Yes vs. No) & $<0.001$ & $2.120(1.345-3.339)$ & 0.001 \\
\hline PNI & (Yes vs. No) & 0.021 & $1.177(0.645-2.149)$ & 0.595 \\
\hline Adjuvant chemo & (Yes vs. No) & 0.108 & & \\
\hline Adjuvant radio & (Yes vs. No) & 0.489 & & \\
\hline
\end{tabular}


Table 3. Univariate and multivariate analyses for recurrence-free survival in patients who received the adjuvant chemotherapy.

\begin{tabular}{lllll}
\hline Variables & & Univariate & Multivariate & \\
\cline { 3 - 5 } & & P-value & HR $(95 \%$ CI $)$ & P-value \\
\hline Sex & (Male vs. Female) & 0.979 & & \\
Age & ( $\geq 65$ vs. $<65)$ & 0.147 & $2.463(1.160-5.231)$ & 0.019 \\
Location & (Rectum vs. Colon) & 0.241 & & \\
Histology & (WMD vs. Others) & 0.684 & & \\
T & (T3,T4 vs. T1,T2) & 0.206 & & \\
$\mathrm{~N}$ & (N1,N2 vs. N0) & 0.069 & $4.362(1.013-18.788)$ & 0.048 \\
CD8 & (High vs. Low) & 0.353 & & \\
STING & (High vs. Low) & 0.026 & $0.423(0.197-0.908)$ & 0.027 \\
LVI & (Yes vs. No) & 0.137 & $2.682(1.259-6.714)$ & 0.011 \\
PNI & (Yes vs. No) & 0.399 & & \\
\hline
\end{tabular}

We also explored the potential of STING expression as a predictor for the response to chemotherapy in patients who received adjuvant chemotherapy. In these patients, patients with higher STING expression showed prolonged 5-year RFS rate $(80.1 \%$ vs. $59.8 \%, \mathrm{P}=0.026)$ after chemotherapy compared with those with lower STING expression. Moreover, when we performed multivariate Cox regression analysis for RFS, high STING expression was indeed an independent predictive factor for chemotherapeutic efficacy (Table 3 ).

\section{Intratumoral STING treatment effectively suppresses colon cancer progression and enhanced intratumoral CD8+ effector $\mathrm{T}$ cells}

In order to confirm whether STING is a valid therapeutic target in $\mathrm{CRC}$, we treated MC38 colon cancers with the STING agonist, 3'3'-cGAMP. Repeated intratumoral injections of the STING agonist suppressed MC38 tumor growth by 57\% when compared to the control tumors (Figure 2A). Intriguingly, one STING-treated tumor completely regressed after the treatment (Figure 2B). Moreover, the survival of STING-treated mice was longer compared to the control mice (Figure 2C). Histologic analysis of the tumor microenvironment revealed an increase in $\mathrm{CD}^{+}$tumor-infiltrating $\mathrm{T}$ cells in STING-treated tumors (Figure 2D). When cell suspensions of the whole tumor were analyzed using flow cytometry, the $\mathrm{CD} 8{ }^{+}$cytotoxic $\mathrm{T}$ cell fraction exhibited a two-fold increase when compared to control (Figure 2E). Moreover, ICOS and IFN- $\gamma$, which are markers for $\mathrm{T}$ cell activation, were also significantly upregulated in $\mathrm{CD}^{+} \mathrm{T}$ cells after intratumoral STING treatment, indicating the
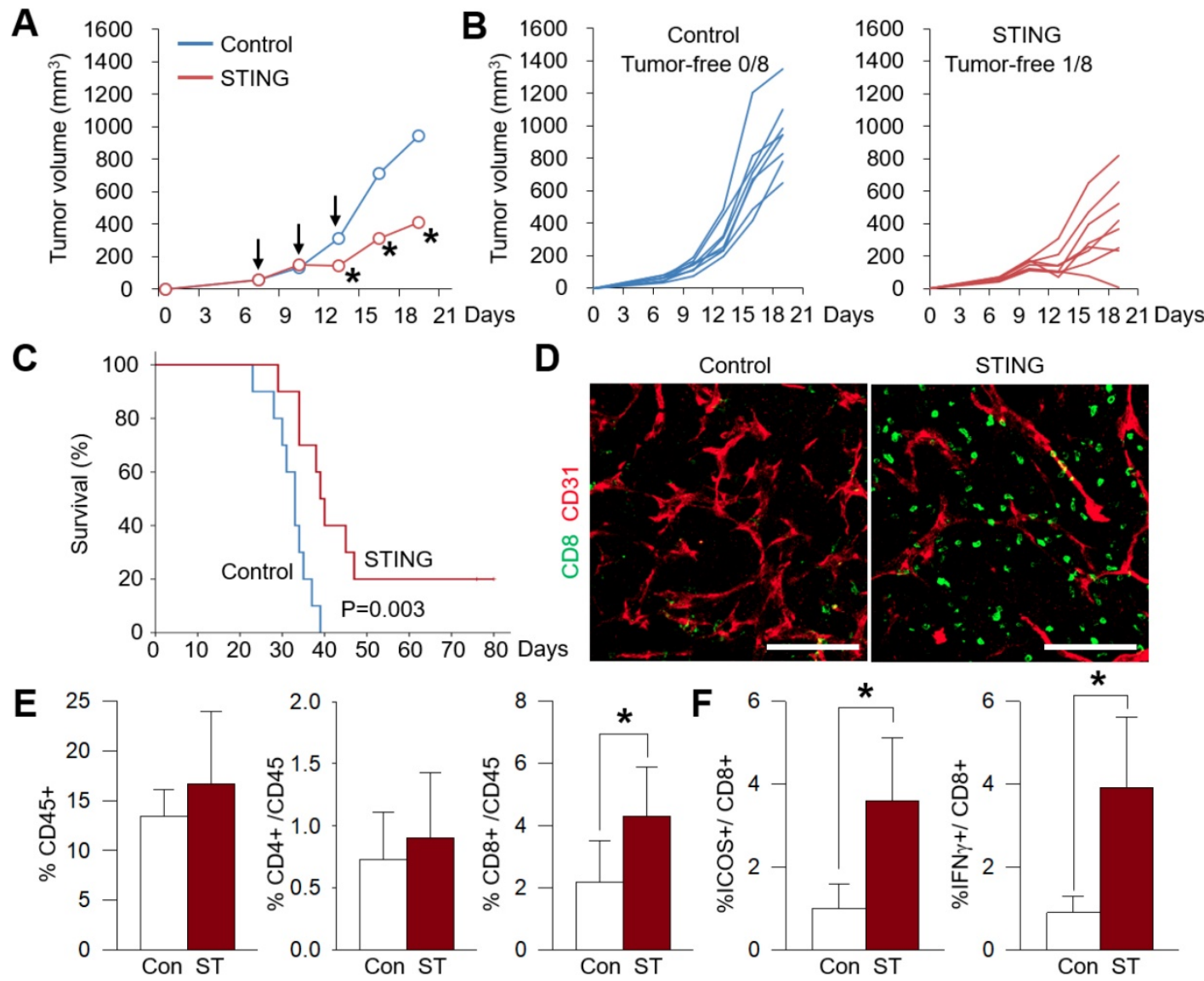

Figure 2. Intratumoral STING activation effectively suppresses colon cancer growth and inflamed colon tumors with activated CD8+ T cells. MC 38 tumors were implanted subcutaneously into B57Cl/6 mice and treated with triple intratumoral injections of STING agonist, 3'3'-cGAMP (10 $\mu$ g), when tumors reached $>50$ mm ${ }^{3}$. Each group, $n=8$. Values are mean \pm STD. ${ }^{*} p<0.05$ versus control. Two-tailed Student's $t$-test was used. (A and B) Average (A) and individual (B) tumor growth curves. (C) Comparison of survival of tumor-bearing mice. (D) Representative images of tumor microenvironment showing CD8 ${ }^{+} \mathrm{T}$ cells (green) and CD31+ tumor blood vessels (red). Scale bars, $100 \mu \mathrm{m}$. (E) Comparison of $\mathrm{CD}^{2} 5^{+}, \mathrm{CD}^{+}$, or $\mathrm{CD} 4+$ cell within tumors. (F) Comparison of $\mathrm{CD}^{+} \mathrm{ICOS} \mathrm{S}^{+}$, and $\mathrm{CD}^{+} \mathrm{GzB}^{+}$cells within tumors. 
activation of intratumoral $\mathrm{CD}^{+} \mathrm{T}$ cells (Figure $2 \mathrm{~F}$ ). Collectively, intratumoral STING treatment was found to effectively suppress colon cancer progression through the enhanced intratumoral trafficking of activated $\mathrm{CD} 8^{+} \mathrm{T}$ cells.

\section{Discussion}

STING signaling plays an important role in establishing anti-cancer immunity. Because DNA normally exists in the nucleus and mitochondria in mammalian cells, its presence in cytoplasm is a danger signal occurring in pathologic condition such as inflammation or cancer. STING is a cytosolic sensor that detects the presence of cytosolic DNA and triggers innate immune system and induces a potent adaptive immune response by upregulating type I IFN genes, enhancing $\mathrm{CD} 8{ }^{+} \mathrm{T}$ cell cross-priming, and strengthening the anti-cancer effector function of $\mathrm{T}$ cells $[15,16,25]$.

Our study elucidated that CRC patients with high STING expression in their tumors had longer survival rates and favorable prognosis compared to those with low STING expression. STING expression remained an independent prognostic biomarker for OS even after being adjusted for tumor stage and CD8 tumor-infiltrating lymphocytes (TILs). Our findings with CRC are in line with previous studies performed on other cancer types, such as hepatocellular carcinoma and gastric cancer [26, 27]. Considering that $\mathrm{CD}^{+}$TILs are highly accumulated in STING-upregulated CRCs and STING is crucial for T-cell cross-priming and activation, intratumoral STING expression could be a useful immune biomarker in identifying T-cell-inflamed cancers.

In this study, we employed an immunogenic colon cancer model, MC38, which is a hypermutated and immunogenic cancer with mismatch repair deficiency to confirm the efficacy of STING-based immunotherapy [28]. Correspondingly, this model showed a good response to STING-based immunotherapy, but the efficacy was not durable, and the rate of complete tumor regression was $\sim 10 \%$. This is because the activation of STING signaling could induce both favorable and unfavorable consequences during immune responses. At first, intratumoral STING activation elicits a strong type I IFN responses and activates $\mathrm{CD} 8^{+} \mathrm{T}$ cells to attack cancer cells. However, when these activated $\mathrm{CD} 8^{+} \mathrm{T}$ cells secretes IFN- $\gamma$, a potent anti-tumor effector cytokine, cancer cells upregulate PD-L1 as a defense mechanism to evade adaptive immune responses [29]. Therefore, simultaneous blockade PD-1 or PD-L1 inhibitor could countervail STING-induced PD-L1 upregulation and enhance the efficacy of STING-based cancer immunotherapy [17]. A few STING agonists, MK-1454
(Merck) and MIW815 (Norvatis), are currently undergoing phase I clinical trials in combination with anti-PD1 immune checkpoint inhibitors in advanced/metastatic solid tumors and lymphomas (NCT03172936, NCT03172936), respectively [30, 31]. Preliminary results show encouraging therapeutic efficacy with acceptable toxicity levels, thereby supporting further clinical development of this novel combination immunotherapy [30].

Although STING is a meaningful prognostic biomarker for various solid tumors, it is still unclear whether the intratumoral STING expression levels are related to the response to immunotherapies. Therefore, the predictive value of STING expression needs further investigation in the ongoing clinical trials.

\section{Conclusion}

In the present study, we revealed an independent prognostic role of STING signaling in CRC and demonstrated that STING-targeted cancer immunotherapy could remodel tumor immune microenvironment of CRC to enhance anti-cancer immunity.

\section{Acknowledgements}

This study was supported by the National Research Foundation of Korea (NRF-2016R1C1B20 14671 to C.K. and 2016R1D1A1B03934012 to H.C.) grant funded by the Korean government (MSIT). This research was also supported by a grant of the Korea Health Technology R\&D Project through the Korea Health Industry Development Institute (KHIDI), funded by the Ministry of Health \& Welfare, Republic of Korea (grant number: HI16C1559).

\section{Competing Interests}

The authors have declared that no competing interest exists.

\section{References}

1. Torre LA, Bray F, Siegel RL, Ferlay J, Lortet-Tieulent J, Jemal A. Global cancer statistics, 2012. CA Cancer J Clin. 2015; 65: 87-108.

2. Kim C, Kim WR, Kim K-Y, Chon HJ, Beom SH, Kim H, et al Predictive nomogram for recurrence of stage i colorectal cancer after curative resection. Clinical Colorectal Cancer. 2018; 17: e513-e8.

3. Bray F, Ferlay J, Soerjomataram I, Siegel RL, Torre LA, Jemal A. Global cancer statistics 2018: GLOBOCAN estimates of incidence and mortality worldwide for 36 cancers in 185 countries. CA: A Cancer Journal for Clinicians. 2018; 68: 394-424.

4. Huang Y, Yuan J, Righi E, Kamoun WS, Ancukiewicz M, Nezivar J, et al. Vascular normalizing doses of antiangiogenic treatment reprogram the immunosuppressive tumor microenvironment and enhance immunotherapy. Proc Natl Acad Sci U S A. 2012; 109: 17561-6.

5. Benci JL, Xu B, Qiu Y, Wu TJ, Dada H, Twyman-Saint Victor C, et al. Tumor Interferon Signaling Regulates a Multigenic Resistance Program to Immune Checkpoint Blockade. Cell. 2016; 167: 1540-54 e12.

6. Spaapen RM, Leung MY, Fuertes MB, Kline JP, Zhang L, Zheng Y, et al. Therapeutic activity of high-dose intratumoral IFN- $\beta$ requires direct effect on the tumor vasculature. The Journal of Immunology. 2014: 1401109 . 
7. Caux C, Ramos RN, Prendergast GC, Bendriss-Vermare N, Ménétrier-Caux C. A milestone review on how macrophages affect tumor growth. Cancer research. 2016; 76: 6439-42.

8. Chon HJ, Lee WS, Yang H, Kong SJ, Lee NK, Moon ES, et al. Tumor Microenvironment Remodeling by Intratumoral Oncolytic Vaccinia Virus Enhances the Efficacy of Immune-Checkpoint Blockade. Clinical Cancer Research. 2019; 25: 1612-23.

9. Albini A, Bruno A, Noonan DM, Mortara L. Contribution to Tumor Angiogenesis From innate immune Cells within the Tumor Microenvironment: implications for immunotherapy. Frontiers in immunology. 2018; 9: 527.

10. Leblond MM, Gérault AN, Corroyer-Dulmont A, MacKenzie ET, Petit E, Bernaudin $\mathrm{M}$, et al. Hypoxia induces macrophage polarization and re-education toward an M2 phenotype in U87 and U251 glioblastoma models. Oncoimmunology. 2016; 5: e1056442.

11. Chen Q, Sun L, Chen ZJ. Regulation and function of the cGAS-STING pathway of cytosolic DNA sensing. Nature immunology. 2016; 17: 1142.

12. Park J-S, Kim I-K, Han S, Park I, Kim C, Bae J, et al. Normalization of tumor vessels by Tie2 activation and Ang2 inhibition enhances drug delivery and produces a favorable tumor microenvironment. Cancer Cell. 2016; 30: 953-67.

13. Ishikawa $\mathrm{H}, \mathrm{Ma} \mathrm{Z}$, Barber GN. STING regulates intracellular DNA-mediated, type I interferon-dependent innate immunity. Nature. 2009; 461: 788.

14. Vargas TR, Benoit-Lizon I, Apetoh L. Rationale for stimulator of interferon genes-targeted cancer immunotherapy. European Journal of Cancer. 2017; 75: $86-97$.

15. Corrales L, Gajewski TF. Molecular Pathways: Targeting the Stimulator of Interferon Genes (STING) in the Immunotherapy of Cancer. Clinical Cancer Research. 2015; 21: 4774-9.

16. Barber GN. STING: infection, inflammation and cancer. Nature Reviews Immunology. 2015; 15: 760-70.

17. Yang H, Lee WS, Kong SJ, Kim CG, Kim JH, Chang SK, et al. STING activation reprograms tumor vasculatures and synergizes with VEGFR2 blockade. The Journal of Clinical Investigation. 2019

18. Kammertoens T, Friese C, Arina A, Idel C, Briesemeister D, Rothe M, et al. Tumour ischaemia by interferon- $\gamma$ resembles physiological blood vessel regression. Nature. 2017; 545: 98.

19. Terawaki S, Chikuma S, Shibayama S, Hayashi T, Yoshida T, Okazaki T, et al. IFN-a directly promotes programmed cell death-1 transcription and limits the duration of T cell-mediated immunity. The Journal of Immunology. 2011; 186: 2772-9.

20. Mandai M, Hamanishi J, Abiko K, Matsumura N, Baba T, Konishi I. Dual faces of IFN- $\gamma$ in cancer progression: a role of PD-L1 induction in the determination of pro-and anti-tumor immunity. Clinical Cancer Research. 2016: clincanres. 0224.2016.

21. Zhu Q, Man SM, Gurung P, Liu Z, Vogel P, Lamkanfi M, et al. Cutting edge: STING mediates protection against colorectal tumorigenesis by governing the magnitude of intestinal inflammation. The Journal of Immunology. 2014: 1402051.

22. Xia T, Konno H, Ahn J, Barber GN. Deregulation of STING Signaling in Colorectal Carcinoma Constrains DNA Damage Responses and Correlates With Tumorigenesis. Cell Reports. 2016; 14: 282-97.

23. Compton CC, Fielding LP, Burgart LJ, Conley B, Cooper HS, Hamilton SR, et al. Prognostic factors in colorectal cancer. College of American Pathologists Consensus Statement 1999. Arch Pathol Lab Med. 2000; 124: 979-94.

24. Yang H, Kim C, Kim M-J, Schwendener RA, Alitalo K, Heston W, et al. Soluble vascular endothelial growth factor receptor-3 suppresses lymphangiogenesis and lymphatic metastasis in bladder cancer. Molecular Cancer. 2011; 10: 36.

25. Rivera Vargas T, Benoit-Lizon I, Apetoh L. Rationale for stimulator of interferon genes-targeted cancer immunotherapy. Eur J Cancer. 2017; 75: 86-97.

26. Bu Y, Liu F, Jia QA, Yu SN. Decreased Expression of TMEM173 Predicts Poor Prognosis in Patients with Hepatocellular Carcinoma. PLoS One. 2016; 11: e0165681.

27. Song S, Peng P, Tang Z, Zhao J, Wu W, Li H, et al. Decreased expression of STING predicts poor prognosis in patients with gastric cancer. Sci Rep. 2017; 7: 39858.

28. Efremova M, Rieder D, Klepsch V, Charoentong P, Finotello F, Hackl H, et al. Targeting immune checkpoints potentiates immunoediting and changes the dynamics of tumor evolution. Nature Communications. 2018; 9: 32.

29. Fu J, Kanne DB, Leong M, Glickman LH, McWhirter SM, Lemmens E, et al. STING agonist formulated cancer vaccines can cure established tumors resistant to PD-1 blockade. Sci Transl Med. 2015; 7: 283 ra52.

30. Harrington $K$, Brody J, Ingham M, Strauss J, Cemerski S, Wang M, et al. LBA15 Preliminary results of the first-in-human (FIH) study of MK-1454, an agonist of stimulator of interferon genes (STING), as monotherapy or in combination with pembrolizumab (pembro) in patients with advanced solid tumors or lymphomas. Annals of Oncology. 2018; 29: mdy424. 015.

31. Wang Y, Deng W, Li N, Neri S, Sharma A, Jiang W, et al. Combining Immunotherapy and Radiotherapy for Cancer Treatment: Current Challenges and Future Directions. Frontiers in pharmacology. 2018; 9: 185. 